\title{
Clinical anatomy of the splanchnic nerves
}

\begin{abstract}
Splanchnic nerves are bilateral visceral autonomic nerves. The thoracic, lumbar and sacral splanchnic nerves are sympathetic in function while the pelvic splanchnic nerves are parasympathetic. These nerves have connections to the celiac, aortic, mesenteric, hypogastric and pelvic plexuses. They control the functions of the gut and pelvic organs. Splanchnicectomies are sometime performed to alleviate intractable abdominal and pelvic pains.
\end{abstract}

Keywords: splanchnic nerves, thoracic, lumbar, sacral, pelvic, nervi erigentes, splanchnicectomy
Volume 5 Issue 2 - 2018

\author{
Heshmat SW Haroun \\ Department of Anatomy and Embryology, Cairo University, \\ Egypt
}

Correspondence: Heshmat SW Haroun, Professor of Anatomy and Embryology, Faculty of Medicine, Cairo University, Egypt, Email heshmatsabet@gmail.com,

heshmat.haroun@kasralainy.edu.eg.com

Received: July 02, 2017 | Published: March 13, 2018

\section{Introduction}

The splanchnic nerves are bilateral autonomic nerves that supply abdominal and pelvic viscera. They are constituted of motor nerve fibers going to the internal organs (visceral efferent fibers) and sensory nerve fibers coming from these organs (visceral afferent fibers). On each side of the human body, they include the thoracic splanchnic nerves (greater, lesser and least or lowest), lumbar splanchnic nerve, sacral splanchnic nerve and pelvic splanchnic nerve (nervus erigentis). All splanchnic nerves carry preganglionic (presynaptic) sympathetic fibers except for the pelvic splanchnic nerves that carry preganglionic parasympathetic fibers. Splanchnicectomy is the procedure used mainly for the control of intractable visceral pain. Detailed knowledge of the anatomy and variations of the splanchnic nerves is mandatory for proper performance of splanchnicectomies (Figure 1).

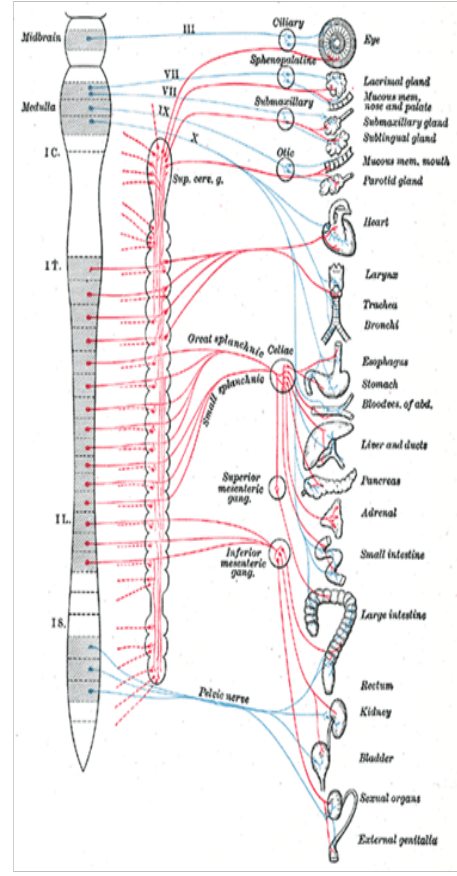

Figure I The autonomic nervous system.

\section{The thoracic splanchnic nerves}

The thoracic splanchnic nerves are made of the medial branches of the lower 7-8 thoracic sympathetic ganglia. Their formation and course are highly variable along their intrathoracic and subdiaphragmatic portions. The greater splanchnic nerve (GSN) is the highest in position of the three nerves and it receives branches from T5-T8 thoracic sympathetic ganglia, the lesser splanchnic nerve (LSN) lies below the greater one and receives branches from T9 and T10 sympathetic ganglia, and the least splanchnic nerve (ISN) is the lowest nerve receiving branches from T11 and/or T12 ganglia. ${ }^{1}$ In a post-mortem study on human posterior thoracic walls, the GSN was formed by branches from T4-T11 ganglia and the commonest type was formed by contributions from T5-T9 ganglia. The LSN was formed by branches from T8-T12 ganglia and the commonest type by branches from T10 and T11 ganglia. The 1SN was composed of branches from T10-L1 ganglia and the commones type by branches from T11 and/or T12 ganglia. ${ }^{2}$ In embalmed South Indian cadavers, the stellate ganglion was bilaterally present in 4 and unilaterally in 15 out of 31 cadavers studied. The highest origin of the GSN was T4 ganglion while its lowest origin was T11 ganglion. The LSN commonly arose from T11 ganglion and the ISN commonly from T12 ganglion. ${ }^{3}$ Adequate information of the anatomical variability of the thoracic sympathetic chain and splanchnic nerves is important for the success of subdiaphragmatic neuroablative surgical procedures to relieve chronic abdominal pain.

Using a special stain and an image-analyser had revealed a decrease in the transverse area and perimeter of the unmyelinated axons the human LSN with the advance of age. ${ }^{4}$ On the contrary, there was no correlation between age and the number of axons of the human GSN but the mean area and perimeter of axons were increased with age. This axon hypertrophy in the GSN was assumed to be a sort of compensation for the hypofunctions in the nervous control of abdominal organs. ${ }^{5}$

Most of the anatomy illustrations demonstrate the three thoracic splanchnic nerves piercing the diaphragmatic crura along three separate superoinferior sites. However, a cadaveric human study has revealed that these three nerves most commonly pierce each diaphragmatic crus through a single location from which the three nerves then diverge to reach their destinations. ${ }^{6}$ 
The GSN and LSN terminate into the celiac ganglion while the ISN into the renal ganglion (behind the renal vessels). The celiac ganglion is a semilunar structure of small ganglionic masses connected together by nerve fibers. It lies on the crus of the diaphragm, on each side of the celiac arterial trunk. The middle suprarenal artery traverses the celiac ganglion and the right ganglion lies behind the inferior vena cava. The thoracic splanchnic nerves and celiac ganglia play an essential role in pain management for upper abdominal disorders, particularly chronic pancreatitis and pancreatic cancer. ${ }^{7}$ Splanchnic nerve dissection, splanchnic nerve thermocoagulation, transhiatal bilateral splanchnicectomy and celiac plexus block are options for splanchnicectomy in management of upper abdominal pain. Thoracoscopic splanchnic denervation (TSD) for chronic pancreatitis pain resulted in fewer patients on opioids and overall increases in pain thresholds. ${ }^{8,9}$

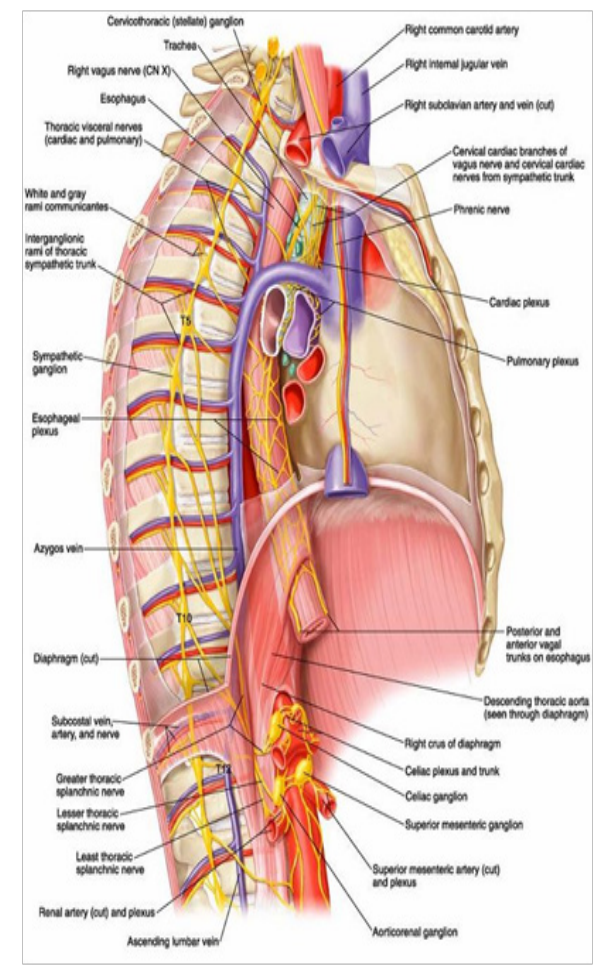

Figure 2 The thoracic splanchnic nerves.

The vascular relations of the right GSN trunk to the azygos vein particularly at its constitutive tributaries: ascending lumbar and subcostal veins and to the thoracic duct in the posterior mediastinum were investigated in human cadavers. The average distances separating the right GSN from the azygos vein and the thoracic duct were $5.7 \mathrm{~mm}$ and $11.2 \mathrm{~mm}$ respectively. These great venous and lymphatic vessels related to the GSN have to be essentially respected at the time of celioscopic splanchnicectomy to avoid the risk of hemorrhage. ${ }^{10}$

In adult pigs, there were sex and laterality differences of the thoracic splanchnic nerves. The GSN was present in all animals included in the study while the LSN was present in $87.5 \%$ of animals on the right side and $75 \%$ on the left side. On the other hand, the ISN was present in $62.5 \%$ of animals on the right side and in $37.5 \%$ on the left side. The right thoracic splanchnic nerves were more cranial in origin, longer and larger than the left nerves. No significant sex differences were determined. ${ }^{11}$ In adult New Zealand rabbits there were no significant sex-differences in the anatomy of the thoracic splanchnic nerves. The GSN and LSN were present in all rabbits while the ISN was found on the right side in $50 \%$ and on the left side in $75 \%$ of studied rabbits. In addition, there were significant laterality differences regarding the origin and structure of these nerves even in the same rabbit. ${ }^{12}$

In Sprague Dawley rats, celiac ganglionectomy (CGX) through surgical removal of the celiac ganglionic plexus was performed with consequent significant reduction of the norepinephrine concentrations in the entire splanchnic area and the mesenteric vessels. There was an abolishment of vasoconstriction of the mesenteric vessels in response to sympathetic nerve stimulation. These effects of CGX were mostly reversible as significant regeneration of sympathetic nerves in some organs was detected at five weeks after surgery. ${ }^{13}$ In the same animal species, stimulation of the superior common splanchnic nerve (innervating the celiac ganglia) had led to decreased food intake, increased metabolic rate, increased lean-to-fat ratio and improved body composition. ${ }^{14,15}$

In immune challenges, the brain influences the immune function through a powerful neural reflex that suppresses the release of inflammatory factors (Figure 2). The efferent motor limb of this neural reflex lies in the splanchnic nerves and not in the vagi. ${ }^{16}$

\section{The lumbar splanchnic nerve}

The lumbar splanchnic nerve, one on each side of the body, arises from the upper two ganglia of the lumbar part of the sympathetic chain (L1\&L2). Its preganglionic sympathetic fibers run medially and downwards to join the aortic plexus where they synapse in the ganglia there and then the postganglionic fibers are distributed to the vessels, smooth muscles and glands of the hindgut and pelvic viscera.

The aortic plexus overlies the front and sides of the abdominal aorta between the origins of the superior and inferior mesenteric arteries. There is general agreement regarding constitution of the aortic plexus of both pre- and post-ganglionic nerve fibers. It is essentially concerned with the sympathetic supply of the hingut, pelvic and urogenital organs. In post-mortem human male specimens, four distinct sympathetic ganglia within the aortic plexus were identified: the right and left spermatic ganglia, the inferior mesenteric ganglion and the prehypogastric ganglion. The spermatic ganglia received L1 presynaptic nerves whereas the inferior mesenteric and prehypogastric ganglia received left and right $\mathrm{L} 2$ presynaptic nerves. These delicate nerves of the aortic plexus are liable to accidental iatrogenic damage during retroperitoneal surgeries; thus threatening male fertility and sympathetic functions of the supplied organs. ${ }^{17}$ The aortic plexus is connected to the superior hypogastric plexus that lies on the bifurcation of the abdominal aorta. Branches of the lumbar and sacral sympathetic chains form the superior hypogastric plexus. At the sacral promontory, this plexus divides into right and left inferior hypogastric (pelvic) plexuses from which nerve fibers spread out bilaterally to the pelvic vessels and organs. ${ }^{18}$

In an experimental study on adult male dogs, each of the canine thoracolumbar splanchnic nerves consisted of two groups of nerve fibers. One group, designated as the intermesenteric splanchnic nerve, arose from the thoracic and L1 sympathetic ganglia, and reached the caudal mesenteric plexus along the anterior wall of the aorta. The other group, designated as the lumbar splanchnic nerve, branched from L2-L5 sympathetic ganglia, and reached the caudal mesenteric plexus along the posterior aspects of both spermatic arteries. Bladder neck closure during ejaculation was observed to occur on electrical stimulation of the lumbar splanchnic nerves and not the intermesenteric or pelvic splanchnic nerves. ${ }^{19}$ 


\section{The sacral and pelvic splanchnic nerves}

The sacral splanchnic nerve, on each side, connects the sacral part of the sympathetic trunk (S1 ganglion) to the inferior hypogasric (pelvic) plexus. Its preganglionic sympathetic fibers ascend from the inferior to the superior hypogastric plexuses then to the aortic and inferior mesenteric plexuses (where they relay), then they go to innervate the hindgut. From each pelvic plexus, sacral splanchnic fibers also supply the pelvic vessels and organs.

Laparoscopic studies of the sacral nerve roots in women revealed delicate fibers emerging from the sacral roots S2, S3 and probably S4 to form the pelvic splanchnic nerve (PSN) on each side. ${ }^{20-22}$ Another laparoscopic sudy in adult male cadavers reported that the PSN (or nervus erigentis) arose from the ventral primary rami of the second, third, and oftenly the fourth sacral nerves; providing preganglionic parasympathetic innervation to the hindgut. The sacral splanchnic nerves emerged from the sacral sympathetic trunks, joined the pelvic splanchnic nerves and then both joined the right and left inferior hypogastric (pelvic) plexuses. ${ }^{23}$

In patients undergoing conventional PSN-sparing radical hysterectomy for cancer cervix uteri, intraoperative electrical stimulation (IES) of the roots of the PSN is recommended. Bladder function has also to be assessed by urodynamic study (UDS) before and after surgery (Figure 3). It is mentioned that IES of PSN roots while monitoring intravesical pressure during radical hysterectomy represents a simple and useful technique for the prediction of postoperative bladder function. ${ }^{24}$

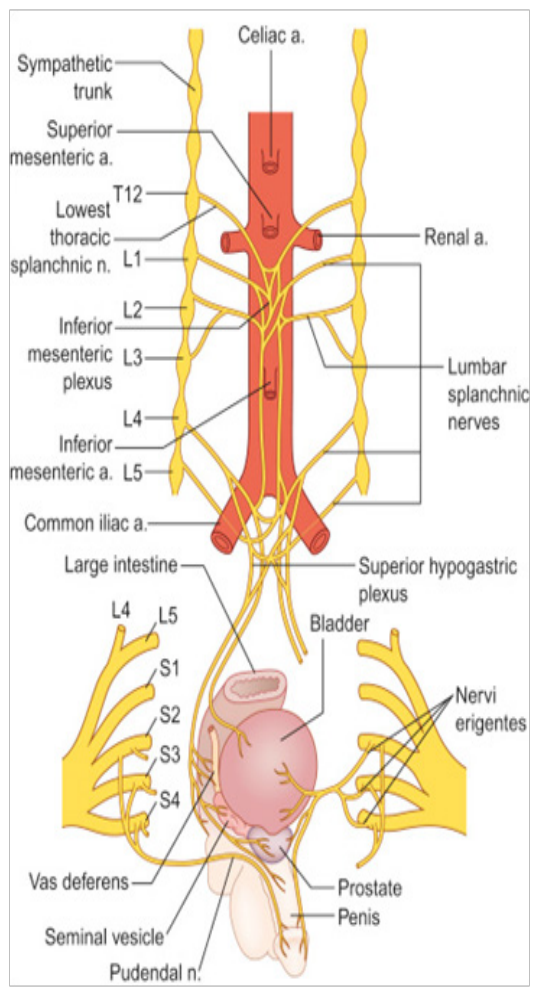

Figure 3 The lumbar, sacral and pelvic splanchnic nerves.

\section{Acknowledgements}

None.

\section{Conflict of interest}

The author declares no conflict of interest.

\section{References}

1. Loukas M, Klaassen Z, Merbs W, et al. A review of the thoracic splanchnic nerves and celiac ganglia. Clin Anat. 2010;23(5):512-522.

2. Yang HJ, Gil YC, Lee WJ, et al. Anatomy of thoracic splanchnic nerves for surgical resection. Clin Anat. 2008;21(2):171-177.

3. Kommuru H, Jothi S, Bapuji P, et al. Thoracic part of sympathetic chain and its branching pattern variations in South Indian cadavers. J Clin Diagn Res. 2014;8(12):AC09-12.

4. Suzuki K, Shiraishi N, Goto N, et al. Unmyelinated nerve fibre analysis of the human lesser splanchnic nerve. Okajimas Folia Anat Jpn. 2000;76(6):285-290.

5. Tanaka J, Goto N, Nagai Y, et al. Myelinated nerve fiber analysis of the human greater splanchnic nerve. Okajimas Folia Anat Jpn. 1999;76(23): $117-121$

6. Gest TR, Hildebrandt S. The pattern of the thoracic splanchnic nerves as they pass through the diaphragm. Clin Anat. 2009;22(7):809-814.

7. Moore KL, Agur AM. Essential Clinical Anatomy. 3rd ed. USA: Lippincott, 2007:181.

8. Buscher HC, van Goor H, Wilder-Smith OH. Effect of thoracoscopic splanchnic denervation on pain processing in chronic pancreatitis patients. Eur J Pain. 2007;11(4):437-443.

9. Masuda T, Kuramoto M, Shimada S, et al. Splanchnicectomy for pancreatic cancer pain. Biomed Res Int. 2014;2014:941726.

10. Ndoye JM, Hamel O, Hamel A, et al. Vascular relationships of the right great splanchnic nerve in the thorax. Morphologie. 2015;99(327):125-131.

11. Orhan O, Duzler A. Anatomy of the thoracic splanchnic nerves in pigs. Vet Res Commun. 2007;31(3):237-243.

12. Duzler A, Dursun N, Cengelci A, et al. The origin and course of the greater, lesser and least thoracic splanchnic nerves in New Zealand rabbits. Anat Histol Embryol. 2003;32(3):183-186.

13. Li M, Galligan J, Wang D, et al. The effects of celiac ganglionectomy on sympathetic innervation to the splanchnic organs in the rat. Auton Neurosci. 2010;154(1-2):66-73.

14. Wu X, McLaughlin L, Polk JP, et al. A pilot study to evaluate the effect of splanchnic nerve stimulation on body composition and food intake in rats. Obes Surg. 2009;19(11):1581-1585.

15. Zheng J, Sonnier T, Vase A, et al. A less invasive surgical approach for splanchnic nerve stimulation to treat obesity. Obes Surg. 2012;22(11):1783-1784.

16. Martelli D, Yao ST, McKinley MJ, et al. Neural control of inflammation by the greater splanchnic nerves. Temperature (Austin). 2014;1(1):14-15.

17. Beveridge TS, Johnson M, Power A, et al. Anatomy of the nerves and ganglia of the aortic plexus in males. $J$ Anat. 2015;226(1):93-103.

18. Shiozawa T, Huebner M, Hirt B, et al. Nerve-preserving sacrocolpopexy: anatomical study and surgical approach. Eur J Obstet Gynecol Reprod Biol. 2010;152(1):103-107.

19. Ando M, Kihara K, Sato K, et al. Regulation of the bladder neck closure by lumbar splanchnic nerves at ejaculation in the dog. Neurourol Urodyn. 1993;12(1):91-98.

20. Zanatta A, Rosin MM, Machado RL, et al. (2014) Laparoscopic dissection and anatomy of sacral nerve roots and pelvic splanchnic nerves. J Minim Invasive Gynecol. 21(6):982-983. 
21. Possover M, Chiantera V, Baekelandt J. Anatomy of the sacral roots and the pelvic splanchnic nerves in women using the LANN technique. Surg Laparosc Endosc Percutan Tech. 2007;17(6):508-510.

22. Lemos N, Souza C, Marques RM, et al. Laparoscopic anatomy of the autonomic nerves of the pelvis and the concept of nerve-sparing surgery by direct visualization of autonomic nerve bundles. Fertil Steril. 2015;104(5):e11-12.
23. Huang J, Zheng Z, Wei H, et al. A comparative study of the laparoscopic appearance and anatomy of the autonomic nervous in normal males. Zhonghua Wai Ke Za Zhi. 2014;52(7):500-503.

24. Katahira A, Niikura H, Kaiho Y, et al. Intraoperative electrical stimulation of the pelvic splanchnic nerves during nerve-sparing radical hysterectomy. Gynecol Oncol. 2005;98(3):462-466. 